\title{
Modifiable Risk Factors Associated with Clearance of Type-Specific Cervical Human Papillomavirus Infections in a Cohort of University Students
}

\author{
Harriet Richardson, ${ }^{1,2}$ Michal Abrahamowicz, ${ }^{2}$ Pierre-Paul Tellier, ${ }^{3}$ Gail Kelsall, ${ }^{1,3}$ \\ Roxane du Berger, ${ }^{2}$ Alex Ferenczy, ${ }^{1,4}$ François Coutlée, ${ }^{5}$ and Eduardo L. Franco ${ }^{1,2}$ \\ Departments of ${ }^{1}$ Oncology, ${ }^{2}$ Epidemiology and Biostatistics, ${ }^{3}$ Family Medicine, and ${ }^{4}$ Pathology, McGill University and \\ ${ }^{5}$ Laboratoire de Virologie Moleculaire, Centre de Recherche et Departement de Microbiologie et Infectiologie, \\ Hôpital Notre-Dame du Centre Hospitalier de l'Université de Montréal, Montreal, Quebec, Canada
}

\begin{abstract}
Background: Previous findings regarding risk factors for human papillomavirus (HPV) persistence, other than viral determinants, identified from prospective cohort studies have been inconsistent in part because study designs have differed with respect to differing HPV detection methods and varying lengths of follow-up time. Therefore, the objectives of this study were to continue the search for epidemiologic risk factors of persistent cervical HPV infections and determine what behaviors differed between those women with transient HPV infections and those women who cannot clear their type-specific HPV infections. Methods: Female university students $(n=621)$ in Montreal were followed for 24 months at 6-month intervals. At each visit, a cervical cell specimen was collected. HPV DNA was detected using the MY09/MY11 PCR protocol and 27 HPV genotypes were identified by the line blot assay
\end{abstract}

(Roche Molecular Systems, Inc., Alameda, CA). Proportional hazards regression was used to estimate the crude and adjusted hazard ratios of clearing a type-specific highrisk $(n=222)$ or low-risk $(n=105)$ HPV infection over time according to specific baseline and time-dependent covariates.

Results: Daily consumption of vegetables seemed to increase the rate of HPV clearance independent of type. The use of tampons was associated with a reduced rate of high-risk HPV clearance, whereas regular condom use was associated with an increased rate of low-risk HPV clearance only.

Conclusion: Some proactive measures can be taken to increase the rate of HPV clearance, and there may be some differences between the sets of predictors of low-risk and high-risk HPV clearance. (Cancer Epidemiol Biomarkers Prev 2005;14(5):1149-56)

\section{Introduction}

The identification of certain sexually acquired human papillomavirus (HPV) infections as the necessary etiologic agent for cervical cancer $(1,2)$ has helped to explain many of the classic risk factors originally identified for cervical cancer, such as age at first intercourse and number of lifetime partners (3). However, given that the evidence from longitudinal cohort studies show that most HPV infections are transient and only women who harbor a persistent HPV infection are likely to develop a cervical squamous intraepithelial lesion (SIL; refs. 4-6), other factors are likely to influence the natural history of HPV infections (7).

Viral determinants, including HPV type and viral load, have been identified as markers of HPV persistence or cervical neoplasia (7-9). Nonetheless, environmental risk factors, some of which are modifiable, are also thought to play a role in cervical carcinogenesis (10). Recent epidemiologic studies have shown that the cofactors most consistently identified in the etiology of cervical neoplasia, after adjusting for the presence of HPV, include high parity and long-term oral contraceptive use (11-14), smoking (15-17), and a coinfection with another sexually transmitted disease $(18,19)$.

Received 3/25/04; revised 6/7/04; accepted 3/7/05.

Grant support: Canadian Institutes of Health Research grant MT-13649, Canadian Institutes of Health Research doctoral scholarship (H. Richardson), Health Scientist Award (M. Abrahamowicz), and Distinguished Scientist Award (E.L. Franco), and Fonds de la Recherche en Sante du Quebec Chercheur Boursier National (F. Coutlée).

The costs of publication of this article were defrayed in part by the payment of page charges. This article must therefore be hereby marked advertisement in accordance with 18 U.S.C. Section 1734 solely to indicate this fact.

Requests for reprints: Eduardo L. Franco, Department of Oncology, McGill University, 546 Pine Avenue West, Montreal, Quebec, Canada H2W 1S6. Phone: 514-398-8014; Fax: 613-398-5002. E-mail: eduardo.franco@mcgill.ca

Copyright (c) 2005 American Association for Cancer Research.
However, it is not clear at which biological stage in HPV carcinogenesis these cofactors are most influential, and results from prospective cohort studies that have attempted to evaluate the effect of hormone contraceptives, diet, alcohol, and tobacco consumption on HPV persistence have been inconsistent $(8,20-22)$. Furthermore, the definition of HPV persistence has varied significantly between studies, and study designs have differed with respect to differing HPV detection methods and varying lengths of follow-up time (5, $8,20-23)$. Consequently, comparison of results in the literature can be challenging.

The objectives of this study were to continue the search for epidemiologic determinants of persistent cervical HPV infections in a cohort of young women attending university in Montreal, Canada. This article presents the results from the analysis of determinants of clearance of typespecific high oncogenic risk and low oncogenic risk HPV infections.

\section{Materials and Methods}

Subjects. Female students attending either the McGill University or the Concordia University Health Clinic were invited to participate if they intended to remain in Montreal, Canada for the next 2 years and had not required treatment for cervical disease in the last 12 months. Recruitment was initiated in November 1996 and accrual was completed in December 1998.

Procedures. All eligible women were asked to return to the clinic every 6 months over a period of 2 years for a total of five visits. At each visit, a questionnaire was completed and 
endocervical and ectocervical cells from the uterine cervix were collected with two accelon cervical biosamplers (Medscand, Inc., Hollywood, FL). A Papanicolaou smear was prepared with the first sampler and the remaining cells along with cervical cells collected with a second sampler were used for HPV DNA testing. At enrollment, information from a detailed, self-administered questionnaire was obtained on potential risk factors, such as sociodemographic status, race, diet, smoking history, sexual behavior, reproductive history, contraceptive and medical history, and personal hygiene. An abridged (follow-up) questionnaire designed to measure changes in recent sexual practices and other lifestyle factors was completed at each subsequent visit.

HPV DNA Detection. HPV DNA testing by a PCR in this study has been described previously elsewhere (24). DNA $(5 \mu \mathrm{L})$ purified with the use of QIAamp columns (Qiagen, Inc., Valencia, CA) was first amplified for $\beta$-globin DNA with PC04 and GH20 primers to show the absence of inhibitors and the integrity of processed DNA (25). Specimens that were $\beta$-globin positive were further tested with the L1 consensus HPV primers MY09/MY11 and HMB01 with Amplitag Gold (TaqGold, Perkin-Elmer-Cetus, Norwalk, CT) and with the line blot assay (Roche Molecular Systems, Inc., Alameda, CA) for the detection of 27 genital HPV genotypes $(26,27)$. HPV types were analyzed individually or in groups according to a classification based on their oncogenic potential. High-risk HPV types included those genotypes that are most frequently found in cervical tumors: HPV 16, 18, 31, 33, 35, 39, 45, 51, 52, $56,58,59$, and 68 (1). All other individual types that were identified with the line blot assay were classified as low-risk HPV types: 6, 11, 26, 40, 42, 53, 54, 55, 57, 66, 73, 82, 83, and 84 .

Statistical Methods. Stratified Cox proportional hazards regression (28) was used to estimate the adjusted relative risk of clearance of prevalent and incident high-risk or low-risk HPV infections over time according to specific baseline characteristics and behaviors. Separate analyses were carried out for clearance of type-specific high-risk and low-risk HPV infections. Variables with repeated measures were represented by their most recent value using time-dependent covariates (28).

The proportional hazards assumption was tested based on a flexible generalization of the Cox proportional hazards model (29). The flexible model allows the hazard ratio (HR) for selected exposures to change over time according to an arbitrary function, the shape of which is estimated from the data using a quadratic regression spline with $5 d f$. The 4- $d f$ likelihood ratio test, comparing the fit of the conventional 1-df proportional hazards model and the flexible 5- $d f$ regression spline model, was used to verify the null hypothesis that the HR between those exposed and those not exposed did not change with increasing time since start of infection. All hypotheses were tested at the 0.05 significance level.

Time to an event was defined as the time between the first HPV-positive visit (detected at enrollment or at any follow-up visit) and the first visit when a subject was no longer positive for her longest enduring, type-specific, high-risk or low-risk HPV infection. Subjects with both high-risk and low-risk HPV types at the index visit were assigned to either the high-risk or the low-risk group based on the type with the longest duration. If there was a tie, the index visit was assigned to the high-risk group to make the two groups mutually exclusive. In case of a tie within the high-risk or low-risk group, all longest persisting infections had to have cleared to be assigned "clearance" status. Those women who completed the study without clearing their infection or were lost to follow-up were censored at their last available visit. Similarly, follow-up time was censored at the date of any biopsy for a low-grade SIL $(n=4)$ and/or treatment for a high-grade SIL $(n=4)$.

We wanted to investigate the role of smoking, alcohol consumption, oral contraceptive, and condom use and certain aspects of diet and personal hygiene habits in clearance of HPV infection. These and other variables with at least one category, with $P<0.20$, after controlling for age were included in later multivariable models so that their independent effects could be evaluated. All analyses were done with SAS version 8.0.

\section{Results}

There were 621 women enrolled in the study that completed a questionnaire and consented to HPV DNA testing. The analysis was based on data collected from 2,650 completed visits (mean of 4.3 visits/subject) of which 2,570 (97.6\%) of the cervical specimens were suitable for HPV DNA testing. Women returned every 6 months on average (65\% returning between 5 and 8 months; range, 2-29 months).

A total of 327 (52.7\%) women had a HPV infection detected at one or more visits and $124(38 \%)$ of those women had at least one visit with a coinfection with high-risk and low-risk HPV types. There were 259 women with a high-risk HPV infection and of those, $37(14 \%)$ had a longer persisting low-risk HPV infection and were excluded from the high-risk group, so that $222(86 \%)$ of the women with a high-risk HPV infection were included in the analysis for clearance of high-risk HPV infections. Of those, 91 (48\%) women eventually cleared their infection, whereas 131 remained positive at their last visit or were lost to follow-up and thus were censored. There were 215 women with a low-risk HPV infection, and of those, 110 (51\%) had a longer persisting high-risk HPV infection and were thus excluded from the low-risk group, so that only $105(49 \%)$ of the women with a low-risk type infection were included in the analysis for clearance of low-risk HPV infections. Of those, 59 $(56 \%)$ women cleared their infection, whereas 46 women were censored.

The distribution of risk factors (Table 1) varied significantly between the cohort members from the two participating university clinics. Participants from university clinic 2 had a significantly higher frequency of persistent high-risk or lowrisk HPV infections, when persistence was defined as three or more consecutive visits with the same high-risk type or lowrisk type. The proportional hazards assumption, according to which the HR is constant over time, was confirmed for all variables of interest (data not shown), except the two university clinics. Thus, given the heterogeneity of the two populations and the evidence that the effect of clinic on clearance of HPV was not constant over time, stratified, time-dependent proportional hazards Cox models were used throughout, with a "nointeraction assumption," to adjust for clinic (30).

There was no significant difference between those women lost to follow-up and among those women who completed follow-up concerning their distribution of risk factors and HPV status at baseline (data not shown). Furthermore, there was no consistent relationship between age and HPV clearance rates (Table 2). Despite this, all models were adjusted for age because it was considered a priori as a confounder of most associations we were interested in exploring. Table 2 presents the age-adjusted HRs for highrisk or low-risk HPV clearance according to putative risk factors. The top part of Table 2 shows estimates for baseline variables identified as time-independent variables, whereas the bottom part of Table 2 shows estimates for variables with repeated measures identified as time-dependent variables. Measures of tobacco, alcohol, condom, and oral contraceptive use along with remaining variables, with $P$ s $<0.20$, in the ageadjusted models (Table 2) were assessed in a single multivariable model (Table 2). Cumulative lifetime exposure to tobacco, expressed as "pack-years," and duration of oral contraceptive use in the participant's lifetime were the dimensions of tobacco and oral contraceptive use retained in the final multivariable models (Table 2). 
Table 1. Distribution of baseline characteristics between participants from the two participating university health clinics

\begin{tabular}{|c|c|c|c|}
\hline Baseline characteristics & $\begin{array}{l}\text { Total cohort } \\
(n=621), n(\%)\end{array}$ & $\begin{array}{l}\text { University clinic } 1 \\
(n=421), \%\end{array}$ & $\begin{array}{l}\text { University clinic } 2 \\
(n=200), \%\end{array}$ \\
\hline \multicolumn{4}{|l|}{ Age (y) } \\
\hline $17-20$ & $187(30.1)$ & 41.3 & 6.5 \\
\hline $21-23$ & $228(36.7)$ & 34.4 & 41.5 \\
\hline $24-26$ & $102(16.4)$ & 11.2 & 27.5 \\
\hline $27+$ & $104(16.7)$ & 13.1 & 24.5 \\
\hline Race & \multicolumn{3}{|c|}{$P_{\chi^{2}}<0.001$} \\
\hline White (Canadian/European descent) & $513(82.6)$ & 82.6 & 82.5 \\
\hline Black (African/Caribbean descent) & $62(10.0)$ & 11.9 & 6.0 \\
\hline Hispanic (South American descent) & $29(4.7)$ & 3.6 & 7.0 \\
\hline Asian (South and East Asian descent) & $17(2.7)$ & 1.9 & 4.5 \\
\hline Age at first sex encounter (y) & \multicolumn{3}{|c|}{$P_{\chi^{2}}=0.009$} \\
\hline $19+$ & $174(28.0)$ & 29.2 & 25.5 \\
\hline $16-18$ & $314(50.6)$ & 52.5 & 46.5 \\
\hline$<16$ & $133(21.4)$ & 18.3 & 28.0 \\
\hline No. lifetime sex partners & \multicolumn{3}{|c|}{$P_{\chi^{2}}=0.025$} \\
\hline 1 & 147 (23.7) & 27.6 & 15.5 \\
\hline $2-4$ & $196(31.6)$ & 34.2 & 26.0 \\
\hline $4-9$ & $161(25.9)$ & 25.7 & 26.5 \\
\hline $10+$ & \multirow{2}{*}{\multicolumn{3}{|c|}{$P_{\chi^{2}}<0.001$}} \\
\hline Pack-vears smoked & & & \\
\hline Nonsmoker & $378(60.9)$ & 67.7 & 46.5 \\
\hline$<1$ & $101(16.3)$ & 16.6 & 15.5 \\
\hline $1-2$ & $58(9.3)$ & 7.1 & 14.0 \\
\hline$>2$ & $84(13.5)$ & 8.6 & 24.0 \\
\hline Alcohol consumption (drinks/wk) & \multicolumn{3}{|c|}{$P_{\chi^{2}}<0.001$} \\
\hline 0 & $226(36.4)$ & 39.2 & 30.5 \\
\hline $1-3$ & $179(28.8)$ & 29.2 & 28.0 \\
\hline$>3$ & $216(34.8)$ & 31.6 & 41.5 \\
\hline & \multicolumn{3}{|c|}{$P_{\chi^{2}}=0.035$} \\
\hline $\begin{array}{l}\text { Duration of oral contraceptive use (y) } \\
0\end{array}$ & \multicolumn{3}{|c|}{ Duration of oral contraceptive use (y) } \\
\hline$<1$ & $121(19.5)$ & $\begin{array}{l}20.7 \\
21.6\end{array}$ & $\begin{array}{l}18.5 \\
15.0\end{array}$ \\
\hline $1-5$ & $252(40.6)$ & 40.1 & 41.5 \\
\hline$>5$ & $103(16.6)$ & 12.6 & 25.0 \\
\hline & \multicolumn{3}{|c|}{$P_{\chi^{2}}<0.001$} \\
\hline \multicolumn{4}{|l|}{ Condom use } \\
\hline Sometimes & $\begin{array}{c}54(8.7) \\
208(33.5)\end{array}$ & $\begin{array}{r}8.1 \\
30.9\end{array}$ & $\begin{array}{l}10.0 \\
39.0\end{array}$ \\
\hline Always & $359(57.8)$ & 61.0 & 51.0 \\
\hline & \multicolumn{3}{|c|}{$P_{\chi^{2}}=0.061$} \\
\hline \multicolumn{4}{|l|}{ History of Chlamydia } \\
\hline Never & $588(94.8)$ & 97.1 & 90.0 \\
\hline \multirow{2}{*}{\multicolumn{4}{|c|}{$P_{\chi^{2}}<0.001$}} \\
\hline & & & \\
\hline Never & $537(86.5)$ & 89.8 & 79.5 \\
\hline Ever & $84(13.5)$ & 10.2 & 20.5 \\
\hline Cumulative HPV status & & $P_{\chi^{2}}<$ & \\
\hline HPV negative (at all visits) & $293(47.3)$ & 53.4 & 34.5 \\
\hline HPV positive (at $1+$ visits) & $32(52.7)$ & 46.6 & 65.5 \\
\hline Persistent high-risk HPV & & $P_{\chi^{2}}<$ & \\
\hline Negative or transient & $548(88.2)$ & 90.5 & 83.5 \\
\hline$\geq 3$ consecutive visits high-risk HPV positive & $73(11.8)$ & 9.5 & 16.5 \\
\hline Persistent low-risk HPV & & $P_{\chi^{2}}=$ & \\
\hline Negative or transient & 583 (93.9) & 95.5 & 90.5 \\
\hline$\geq 3$ consecutive visits low-risk HPV positive & $38(6.1)$ & 4.5 & 9.5 \\
\hline Cumulative SU status & & $P_{\chi^{2}}=$ & \\
\hline $\begin{array}{l}\text { Cumulative SIL status } \\
\text { Normal cytology (at all visits) }\end{array}$ & 575 (92.6) & 91.9 & \\
\hline $\begin{array}{l}\text { Normal cytology (at all visits) } \\
\text { SIL (at } \geq 1 \text { visit) }\end{array}$ & $46(7.4)$ & $\begin{array}{r}91.9 \\
8.1\end{array}$ & $\begin{array}{r}94.0 \\
6.0\end{array}$ \\
\hline & & $P_{\chi^{2}}<$ & \\
\hline
\end{tabular}

Ethnicity was associated with clearance of high-risk HPV but not low-risk HPV infections (Table 2). However, although Black, Hispanic, and Asian women seemed to clear their highrisk HPV infections more rapidly than White women, only
Black women were significantly more likely to clear their highrisk HPV infection compared with White women [HR, 2.86; $95 \%$ confidence interval (95\% CI), 1.18-6.95]. Surprisingly, increased alcohol consumption was associated with a 2-fold 
Table 2. Determinants of clearance of type-specific high-risk and low-risk HPV infections: age-adjusted and final multivariable model

Time-independent variables

\begin{tabular}{l} 
\\
\hline Age (y) \\
$17-20$ \\
$21-23$ \\
$24-26$ \\
$27+$ \\
Age at first sex encounter (y) \\
$19+$ \\
$16-18$ \\
$<16$ \\
Ethnicity \\
White (Canadian/ \\
European descent) \\
Black (African/ \\
Caribbean descent) \\
Hispanic (South American \\
descent) \\
Asian (South and East \\
Asian descent) \\
Sanitary products \\
Tampons
\end{tabular}

Sanitary napkins

Sanitary napkins and tampons

Vegetable consumption

(daily servings)

$<1$

$1+$

Duration of oral

contraceptive use (y)

0

$<1$

$1-5$

$>5$

Smoking status

Never

Former

Current

No. cigarettes daily

Nonsmoker

$$
<1
$$$$
1-5
$$

$>5$

Pack-years smoked

Nonsmoker

$<1$

$1-2$

$>2$

Alcohol consumption

(drinks/wk)

0

1-3

$>3$

Oral contraceptive use

Never

Sometimes

Always

Condom use

Never

Sometimes

Always

History of Chlamydia

No

Yes

Wash after sex

Never/rarely
High oncogenic risk $(n=222)$

Age adjusted, Multivariable model, HR $(95 \% \mathrm{CI}) \quad$ HR $(95 \% \mathrm{CI})$

$\begin{array}{ll}\text { Reference } & \text { Reference } \\ 1.1(0.7-1.7) & 1.3(0.7-2.5) \\ 0.6(0.3-1.1) & 0.7(0.3-1.6) \\ 0.8(0.4-1.7) & 0.7(0.3-1.9)\end{array}$

Reference

$0.8(0.5-1.3)$

$0.8(0.5-1.5)$

Reference $^{\dagger}$

Not included

in final model ${ }^{*}$

Reference

$1.8(0.8-3.7)$

$1.9(0.6-6.0)$

$1.2(0.5-6.0)$

Reference $^{\ddagger}$

$3.3(1.7-10.0)$

$2.0(1.1-3.3)$

Reference $^{\ddagger}$

$1.7(1.1-3.3)$

Reference $^{\dagger}$

$1.4(0.8-2.5)$

$0.8(0.5-1.4)$

$0.8(0.4-1.7)$

Reference

$1.0(0.5-2.0)$

$0.7(0.5-1.2)$

Reference $^{\dagger}$

$0.4(0.2-1.0)$

$0.9(0.5-1.8)$

0.8 (0.4-1.5)

Reference $^{\dagger}$

$0.8(0.5-1.5)$

$0.6(0.3-1.2)$

0.7 (0.4-1.4)

Reference

$1.1(0.6-1.9)$

$1.0(0.5-1.8)$

Reference

$0.8(0.3-2.2)$

$0.8(0.3-1.3)$

Reference

$0.8(0.5-1.5)$

$1.1(0.6-1.7)$

Reference

$1.4(0.3-5.9)$

Reference

Reference

$5.0(2.5-10.0)$

$2.5(1.4-5.0)$

Reference

2.5 (1.4-5.0)

Reference

$1.7(0.8-3.5)$

$1.2(0.6-2.4)$

$1.0(0.4-2.5)$

Not included

in final model ${ }^{\S}$

Not included

in final model

Reference

$0.7(0.4-1.4)$

$0.5(0.2-1.1)$

$0.6(0.3-1.3)$

Reference

$2.1(1.0-4.5)$

$2.0(1.0-4.9)$

Not included

in final model ${ }^{\S}$

Reference

$0.8(0.4-1.5)$

$1.0(0.6-1.7)$

Not included in final model ${ }^{*}$

Not included
Low oncogenic risk $(n=105)$

Age adjusted, Multivariable model, HR $(95 \%$ CI $) \quad$ HR $(95 \%$ CI $)$

Reference

$0.9(0.5-1.7)$

$0.8(0.4-1.6)$

$0.9(0.4-1.8)$

Reference

$1.4(0.5-3.9)$

$0.9(0.3-2.8)$

$1.3(0.4-4.6)$

Reference

$1.2(0.6-2.1)$

Not included in final model ${ }^{*}$

$0.9(0.4-1.9)$

Reference

$0.7(0.2-2.2)$

Not included in final model ${ }^{*}$

$3.0(0.4-22.3)$

$1.0(0.3-2.7)$

Reference

Not included

$1.0(0.5-2.0)$

$1.3(0.7-2.5)$

Reference $^{\dagger}$

$1.6(0.9-2.5)$

Reference

$2.0(0.9-5.0)$

Reference

$0.7(0.2-2.0)$

$1.0(0.5-2.0)$

$1.4(0.6-3.1)$

Reference

$0.7(0.3-1.8)$

$1.1(0.6-2.1)$

Reference

$1.2(0.6-2.6)$

$1.1(0.5-2.5)$

$1.4(0.6-2.9)$

Reference

$1.3(0.7-2.5)$

$0.5(0.2-1.9)$

$1.1(0.5-2.2)$

Reference

$1.2(0.3-4.2)$

$1.9(0.7-5.5)$

$2.3(0.6-9.2)$

Not included in final model ${ }^{*}$

Reference

$1.4(0.6-3.2)$

$1.9(0.9-4.3)$

Reference

$0.0(0.0-)$

$1.1(0.6-1.9)$

Reference $^{\ddagger}$

$1.2(0.5-2.5)$

$2.3(1.1-4.7)$

Not included

in final model

Not possible to calculate

Reference

$0.8(0.3-1.8)$

$0.3(0.1-1.1)$

$0.7(0.3-1.9)$

Reference

$1.1(0.4-2.7)$

$1.9(0.7-5.3)$

Not included in final model

Reference

$2.3(0.9-5.8)$

$4.2(1.7-10.3)$

Not possible to calculate in final model ${ }^{*}$

Reference

Not included in final model ${ }^{*}$ 
Table 2. Determinants of clearance of type-specific high-risk and low-risk HPV infections: age-adjusted and final multivariable model (Cont'd)

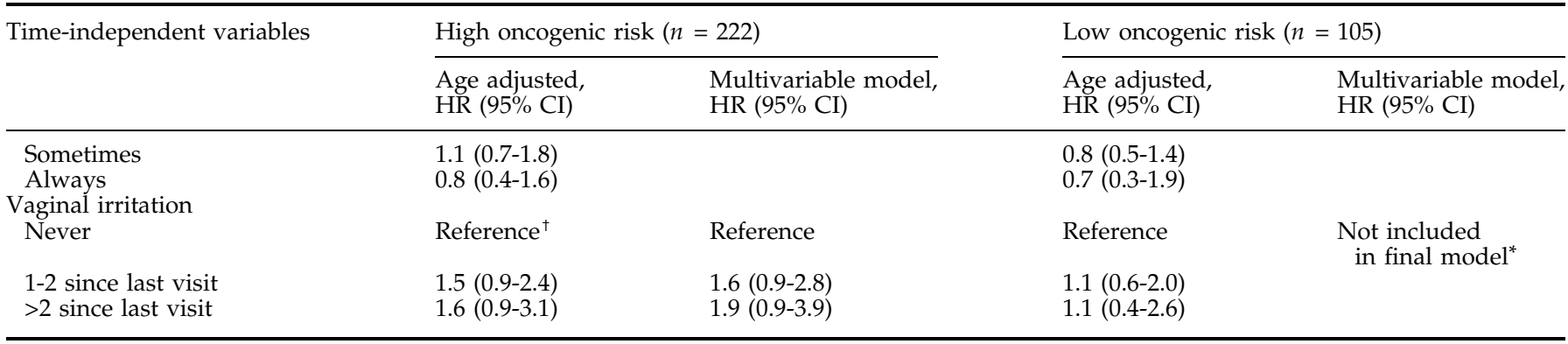

NOTE: All variables were adjusted for each other in the final model.

*These variables were not included in the final model for clearance of high-risk or low-risk infections because of a clear lack of association in earlier models.

$\dagger P<0.20$.

$\ddagger P<0.05$.

§These variables were not included in the final model for clearance because other indices of the same exposure were selected instead.

increased rate of clearance for both high-risk and low-risk HPV infections when adjusted for other predictors of clearance, including tobacco use. However, women who smoked the equivalent of one or two packs of cigarettes per day (e.g., 20 cigarettes per pack) for at least 1 year were less likely to clear their high-risk HPV (HR, 0.5; 95\% CI, 0.2-1.1) or low-risk HPV (HR, 0.3; 95\% CI, 0.1-1.1) infections compared with women who never smoked. Similarly, compared with women who did not eat vegetables daily, women who had one or more daily servings of vegetables (carrots, broccoli, cabbage, salad, or green beans) cleared their high-risk HPV (HR, 2.5; 95\% CI, 1.45.0) or low-risk HPV (HR, 2.0; 95\% CI, 0.9-5.0) infections more rapidly (Table 2 ).

The association between sexually transmitted diseases and HPV persistence could not be well evaluated because there were too few women with a HPV infection and a past or recent sexually transmitted disease, such as Chlamydia trachomatis or a herpes simplex virus-2 infection (Table 2). Barrier methods of contraception were associated with faster clearance of low-risk HPV infections (HR, 4.2; 95\% CI, 1.7-10.3), whereas increased duration of oral contraceptive use was also associated with a faster rate of low-risk HPV clearance but was not statistically significant.

Most interestingly, the use of tampons was a strong predictor of high-risk HPV persistence, and women who used sanitary napkins, exclusively, were five times (HR, 5.0; 95\% CI, 2.5-10.0) more likely to clear their high-risk HPV infection compared with women who used tampons, exclusively.

\section{Discussion}

The viral-host interaction is believed to play a crucial role in HPV persistence. Furthermore, functional differences between oncoproteins of viral HPV subgroups are believed to explain the ability of high-risk HPV types to cause cellular transformation, proliferation, and lesion progression (31-33). For example, estradiol has been shown to stimulate transcription of E6 and E7 gene products of HPV 16 (34). However, it remains to be shown that hormonal stimuli can also influence transcriptional activity of other HPV types, particularly lowrisk types. If there are biological distinctions between the molecular mechanisms of low-risk HPV and high-risk HPVinduced infections, there may also be distinct sets of risk factors for persistence associated with these HPV subgroups. When we did an analysis without grouping type-specific infections into high-risk and low-risk groups, many of the associations that we observed in the stratified (on oncogenic group) analyses disappeared or were diluted (data not shown).
Thus, certain effects may be masked if all type-specific infections are grouped together (35).

The rationale for studying mutually exclusive groups by dividing women with coinfections into the HPV-risk group according to her most persistent type infection was to avoid mixing a preexisting infection and a newly acquired (different type) infection in the definition of persistence (or clearance; ref. 24). We also wanted to study determinants of a woman's most persistent HPV infection, because the evidence suggests that it is the most persistent type-specific infections that best predict progression to high-grade SIL (6) and cervical cancer.

Results from our study suggest that regular condom use is significantly protective against low-risk HPV persistence. Conflicting reports from cross-sectional studies demonstrating protective effects of condom use against low-risk HPV (36) or high-risk HPV infection only $(37,38)$ suggest that condoms may confer varying levels of protection against different HPV types. However, whether this means that genital HPV types have altered biological tropisms and can be found, differentially, on specific anatomic genital sites that are not all protected by a condom still needs to be clarified (37-39).

Although condom use and other barrier contraceptives have not been shown to be associated with HPV persistence in previous studies $(20,23)$, regular use of condoms has been associated with a reduction in risk for high-grade SILs among HPV-positive women in two separate cohorts $(8,12)$. These results are fairly unexpected, because it has generally been thought that if condoms do confer protection it would be further upstream, by preventing transmission of HPV, not lesion development. However, it is possible that, if condoms do protect against acquisition of a new HPV infection, then extended persistent HPV episodes (reinfection with the same type) may be prevented. Another possibility is that, although condoms may not entirely protect against transmission, they may help reduce the viral load of HPV or protect against other sexually transmitted diseases that may interact with HPV and worsen the natural course of infection (12). This hypothesis is further supported with results from a recent clinical trial in the Netherlands that showed condom use, for at least 3 months, helped to promote regression of cervical intraepithelial neoplasia and clearance of HPV among women with cervical intraepithelial neoplasia (40).

The carcinogenic effects of tobacco exposure on the development of cervical disease have been studied extensively in both epidemiologic and experimental studies (41). It has been shown that tobacco exposure affects the host immunosurveillance system against viral infections as shown by reduced markers of immune function in vitro (42). In addition, tobacco-specific nitrosamine 4-(methylnitrosamino)-1-(3pyridyl)-1-butanone has been measured in cervical mucosa 
(43), and there is also preliminary laboratory evidence to suggest that certain HPV-infected cells may be more susceptible to DNA damage from specific tobacco carcinogens (44).

Nonetheless, based on the current literature, it is still difficult to conclude at which stage in the natural history of HPV cervical carcinogenesis tobacco exposure would exert most influence. Although a consistent relationship between tobacco use and in situ or invasive cervical carcinoma has been observed in case-control and cohort studies as reviewed in ref. 41, the association between smoking tobacco and HPV persistence has not been consistently shown in the literature $(5,8,21)$. Nonsmokers were surprisingly more likely to have a persistent HPV infection than current smokers among a cohort of university students in New York followed every 6 months for $>2$ years (8) and among a cohort of middle-aged women in Oregon who returned for one follow-up visit (5). Conversely, Giuliano et al. (21) observed that women who smoked were less likely to clear a HPV infection than nonsmokers among a cohort of women who returned for two follow-up visits over an average of 10 months. Others have not observed any association between tobacco use and HPV persistence (23) orclearance $(20,22)$. However, only one of these studies (21) evaluated the cumulative intensity of tobacco exposure (pack-years).

Cumulative intensity of exposure to tobacco metabolites (often measured in "pack-years") may be a better predictor of genomic damage in squamous epithelial cells and risk of subsequent development of cervical disease (17). However, if exposure to tobacco is also associated with HPV clearance (a rather rapidly evolving event), suppression of the immune response is more likely to be the mechanistic explanation rather then DNA damage (42), and presumably recent and cumulative tobacco exposure would have similar influences on cell-mediated immunity against viral infections. Nonetheless, we observed that women who smoked one or two packs of cigarettes per day for at least 1 year were nearly half as likely to clear their HPV infections then nonsmokers, although the association was marginally nonsignificant, and there was not a clear dose response. However, a dose response with tobacco use may have been hard to observe in this study because of the relatively low levels of tobacco exposure in this young cohort. When we evaluated the effect of recent number of cigarettes smoked or smoking status (never, former, or current) in the final multivariable model, neither variable was associated with HPV clearance (data not shown).

Other factors that may reduce immune function or affect the health of the cervical squamous epithelial environment could include oral contraceptive use $(22,45)$, excessive alcohol consumption $(46,47)$, and dietary patterns deficient in essential micronutrients (48). Although oral contraceptives seem to be a cofactor in cervical carcinogenesis (41), the majority of cohort studies have not observed an association with duration of oral contraceptive use and HPV persistence $(5,8,20)$. In our study, however, we observed that women who used oral contraceptives regularly for at least 1 year cleared their low-risk HPV infections more rapidly then women who did not use oral contraceptives. These findings partially support results from a recent population-based cohort study conducted in Bogota, Colombia (22). Women were followed for at least 5 years and the authors observed that women who had ever used oral contraceptives experienced faster clearance of any type-specific HPV infection compared with women who were nonusers of oral contraceptives (22). However, because oral contraceptive use may be a proxy for monogamous sexual relationships, more epidemiologic and laboratory studies are required to clarify whether oral contraceptives exert a real protective biological effect within the cervical microenvironment or whether our results are confounded by "safe partners."

A limited food frequency questionnaire was completed by our cohort members at enrollment only, and the main goal was to characterize general eating patterns with respect to dairy products and fruits and vegetables that were high in vitamins $\mathrm{C}$ and E, carotenoids, and folate. Two different mechanistic pathways have been proposed to support the protective effects of a healthy diet against cervical carcinogenesis. Antioxidant nutrients, such as vitamins $\mathrm{C}$ and $\mathrm{E}$, lycopene, and carotenoids, may act early in cervical carcinogenesis by helping to prevent cellular and DNA damage from reactive oxygen species caused from exposure to tobacco or cervical inflammation (48). Folate as well as vitamins $B_{12}$ and $B_{6}$ and methionine may also play a role in decreasing viral proliferation through their role in DNA methylation (49).

Despite our crude measurement of dietary patterns, we observed that women who consumed one or more servings of vegetables per day cleared their HPV infections significantly more quickly than women who did not consume vegetables daily. Our results add to the mounting evidence that fresh vegetables may play a protective role against persistent HPV infections. A recent study that measured food intake with a food frequency questionnaire and also measured levels of specific micronutrients in blood samples observed that a higher level of vegetable consumption was associated with a $54 \%$ decrease risk of HPV persistence in a cohort of young women from Arizona (49). Furthermore, lower levels of important micronutrients found in vegetables, such as $\beta$-cryptoxanthin and lutein/zeaxanthin, were associated with increased HPV persistence in the Arizona cohort (50) and similarly among a cohort of women in Brazil (51).

Whereas fruits and vegetables may protect against HPV persistence, excessive alcohol use may increase the risk of HPV persistence by interfering with folate absorption and metabolism (52). Paradoxically, in our study, alcohol use seemed to be associated with a more rapid rate of HPV clearance. These results are not in agreement with other cohort studies among young women who showed that increased alcohol consumption was either associated with HPV persistence (8) or not associated with HPV clearance at all (53). However, $\sim 90 \%$ of the cohort members in our study drank less than one drink per day. Therefore, the effect of heavy drinking on HPV clearance could not be adequately assessed.

Hygienic practices, such as douching and use of tampons during menstruation, have frequently been proposed as explanatory variables for observed differences in HPV prevalence, but associations have been inconsistent $(8,35,38)$. Our data showed that women who used tampons instead of sanitary napkins were significantly less likely to clear their high-risk HPV infection. One other cohort study observed a similar elevated risk for cumulative high-risk HPV among women who used tampons instead of sanitary napkins during menstruation (35). Although the mechanism is currently unknown, it is possible that tampons will serve to spread the viral infection to different locations in the cervix, thereby increasing the area and possibly the severity of the HPV infection. Cervical inflammation has also recently been proposed as a relevant cofactor in HPV carcinogenesis (48) and perhaps the premature removal of tampons may induce some form of epithelial irritation that may facilitate persistence of a high-risk HPV infection. Nonetheless, regardless of the mechanism, if future studies among populations with equally high exposure to tampons reveal similar measures of effect, then discouraging the use of tampons during the course of a HPV infection may prove to be one of the more easily modifiable determinants of rapid HPV clearance.

The observation that Black women from African or Caribbean descent cleared their high-risk HPV infections more rapidly then White women (from European descent) was unexpected and could not be explained by the student's perceived level of their family's financial situation ("difficult," "moderate," or "very comfortable"; data not shown). 
However, White subjects were also more likely to have engaged in sexual activities at an earlier age ( $<16$ years) compared with other women $(P<0.001)$. Age at first intercourse may be associated with latent infections because it could be a proxy for time since first possible exposure to HPV. One recent cohort study observed a slower clearance rate (relative risk, 0.74; 95\% CI, 0.52-1.06) for women who engaged in sexual relationships at age $\leq 15$ years (22). However, we did not observe a clear relationship with age at first intercourse and HPV clearance in our study, and it did not alter the estimates of effect for ethnicity substantially when added to a regression model that only included ethnicity.

There are some limitations in our study that should be addressed. Both prevalent and incident infections were included in our analysis of clearance. One concern with combining prevalent and incident HPV data is that the time at which a woman is first HPV positive cannot be easily approximated for prevalent infections, even if the time of clearance is well approximated, and leads to a form of left censoring. Furthermore, including prevalent cases may introduce a type of survival bias because a greater proportion of the prevalent cases may represent persistent infections compared with the incident cases. However, when we evaluated the association between selected risk factors and clearance rates of incident HPV infections, the HRs did not differ materially from the point estimates presented in Table 2, although the $95 \%$ CIs were wider (data not shown). Therefore, the potential length bias introduced by including prevalent cases in the analysis appeared minimal and it was decided to combine both incident and prevalent infections in the analysis to improve the power of the analyses.

Etiologic studies on HPV persistence have varied widely with respect to length of time between return visits. This difference in time can affect the results as women with a longer time interval between two visits ( $>12$ months) are significantly less likely to have a persistent HPV infection (12). Extreme variation in length of time between visits could also have biased our estimated time to HPV clearance. Although the majority of women in our cohort returned within 6 months of each visit, there was a small proportion of women whose time interval between visits was $>1$ year. If this subgroup of women had differentially cleared their infection at a much earlier date before their return visit date, the average time to clearance could be inflated. However, the association between putative risk factors and the clearance rate of HPV would only be distorted if certain exposure-outcome combinations were associated differentially with time between visits. Given that the participants were unaware of their HPV status until the end of the study, this is an unlikely scenario.

In conclusion, viral characteristics and the host environment (i.e., genetic susceptibility, and immunosuppression) are currently the most important determinants of HPV persistence (7). Therefore, until a vaccine or antiviral medication is available to treat HPV infections, there seems to be little that a physician can do for a HPV-positive patient other than see her regularly for cervical cytology screening. However, our results suggest that there may be some proactive measures that a woman can take to modify her risk for HPV persistence. The cessation of tobacco use, increased vegetable consumption, and use of sanitary napkins instead of tampons during the course of a HPV infection are actions that may potentially lead to a faster rate of HPV clearance.

\section{Acknowledgments}

We thank Suzanne Dumais and the staff at McGill University and Concordia Student Health Services for help with subject accrual and follow-up, Roche Molecular Systems for the generous supply of all the reagents necessary for HPV genotyping, and Helêne Voyer for meticulous laboratory work and HPV testing.

\section{References}

1. Bosch F, Manos M, Muñoz N, et al. Prevalence of human papillomavirus in cervical cancer-a worldwide perspective. J Natl Cancer Inst 1995;87:796 - 802.

2. Walboomers J, Jacobs M, Manos M, et al. Human papillomavirus is a necessary cause of invasive cervical cancer worldwide. J Pathol 1999; 189:12-9.

3. Brinton L. Epidemiology of cervical cancer-overview. In: Muñoz N, Bosch FX, Shah KV, Meheus A, editors. The epidemiology of human papillomavirus and cervical cancer. Lyon: IARC Scientific Publications; 1992. p. 3-22

4. Moscicki A, Palefsky J, Smith G, Siboshski S, Schoolnik G. Variability of human papillomavirus DNA testing in a longitudinal cohort of young women. Obstet Gynecol 1993;82:578-85.

5. Hildesheim A, Schiffman M, Gravitt P, et al. Persistence of type-specific human papillomavirus infection among cytologically normal women. J Infect Dis 1994;169:235-40.

6. Schlecht N, Kulaga S, Robitaille J, et al. Persistent human papillomavirus infection as a predictor of cervical intraepithelial neoplasia. JAMA 2001;286:3106-14.

7. Bosch F, Lorincz A, Muñoz N, Meijer C, Shah K. The causal relation between human papillomavirus and cervical cancer. J Clin Pathol 2002;55:0-21.

8. Ho G, Bierman R, Beardsley L, Chang C, Burk R. Natural history of cervicovaginal papillomavirus infection in young women. N Engl J Med 1998;338:423-8.

9. Lorincz A, Castle P, Sherman M, et al. Viral load of human papillomavirus and risk of CIN3 or cervical cancer. Lancet 2002;360:228-9.

10. Castellsague $X$, Bosch FX, Muñoz N. Environmental co-factors in HPV carcinogenesis. Virus Res 2002;89:191-9.

11. Lacey J, Brinton L, Abbas F, et al. Oral contraceptives as risk factors for cervical adenocarcinomas and squamous cell carcinomas. Cancer Epidemiol Biomarkers Prev 1999;8:1079-85.

12. Hildesheim A, Herrero R, Castle P, et al. HPV co-factors related to the development of cervical cancer: results from a population-based study in Costa Rica. Br J Cancer 2001;84:1219-26.

13. Moreno V, Bosch X, Muñoz N, et al. Effect of oral contraceptives on risk of cervical cancer in women with human papillomavirus infection: the IARC multicentric case-control study. Lancet 2002;359:1085-92.

14. Muñoz N, Franceschi S, Bosetti C, Morreno V, Herrero R, Smith J. Role of parity and human papillomavirus in cervical cancer: the IARC multicentric case-control study. Lancet 2002;359:1093-101.

15. Szarewski A, Cuzick J. Smoking and cervical neoplasia: a review of the evidence. J Epidemiol Biostat 1998;3:229-56.

16. Deacon I, Evans C, Yule R, et al. Sexual behaviour and smoking as determinants of cervical HPV infection and of CIN3 among those infected: a case-control study nested within the Manchester cohort. $\mathrm{Br} \mathrm{J}$ Cancer 2000;88:1565-72.

17. Castle PE, Wacholder S, Lorincz AT, et al. A prospective study of high-grade cervical neoplasia risk among human papillomavirus-infected women. J Natl Cancer Inst 2002;94:1406-14

18. Anttila T, Saikku P, Koskela P, et al. Serotypes of Chlamydia trachomatis and risk of development of cervical squamous cell carcinoma. JAMA 2001;285:47-51.

19. Smith J, Muñoz N, Herrero R, et al. Evidence for Chlamydia trachomatis as an HPV cofactor in the etiology of invasive cervical cancer in Brazil and the Philippines. J Infect Dis 2002;185:324-31.

20. Moscicki A, Shiboski S, Broering J, et al. The natural history of human papillomavirus infection as measured by repeated DNA testing in adolescent and young women. J Pediatr 1998;132:277-84.

21. Giuliano A, Sedjo R, Roe D, et al. Clearance of oncogenic human papillomavirus (HPV) infection: effect of smoking (United States). Cancer Causes Control 2002;13:839-46.

22. Molano M, Van den BA, Plummer M, et al. Determinants of clearance of human papillomavirus infections in Colombian women with normal cytology: a population-based, 5-year follow-up study. Am J Epidemiol 2003;158:486-94.

23. Brisson J, Bairati I, Morin C, et al. Determinants of persistent detection of human papillomavirus DNA in the uterine cervix. J Infect Dis 1996; 173:794-9.

24. Richardson H, Kelsall G, Tellier P, et al. The natural history of type-specific human papillomavirus infections in female university students. Cancer Epidemiol Biomarkers Prev 2003;12:485-90.

25. Bauer H, Ting Y, Greer C, et al. Genital human papillomavirus infection in female university students as determined by a PCR-based method. JAMA 1991;265:472-7.

26. Gravitt P, Peyton C, Apple R, Wheeler C. Genotyping of 27 human papillomavirus types by using L1 consensus PCR products by singlehybridization, reverse line blot detection method. J Clin Microbiol 1998; 36:3020-7.

27. Coutlée F, Gravitt P, Kornegay J, et al. Use of PGMY primers in L1 consensus PCR improves detection of human papillomavirus DNA in genital samples. J Clin Microbiol 2002;40:902-7.

28. Cox D. Regression models and life tables (with discussion). JR Stat Soc Ser B 1972;34:187-220.

29. Abrahamowicz M, MacKenzie T, Esdaile J. Time-dependent hazard ratio: modeling and hypothesis testing with application in lupus nephritis. J Am Stat Assoc 1996;91:1432-9.

30. Kleinbaum D. Survival analysis. New York: Springer-Verlag; 1996. 
31. de Sanjose S, Muñoz N, Bosch F, et al. Sexually transmitted agents and cervical neoplasia in Colombia and Spain. Int J Cancer 1994;56:358-63.

32. Ho G, Burk R, Fleming I, Klein R. Risk for human papillomavirus infection in women with human immunodeficiency virus-induced immunosuppression. Int J Cancer 1994;56:788-92.

33. zur Hausen H. Papillomavirus causing cancer: evasion from host-cell control in early events in carcinogenesis. J Natl Cancer Inst 2000;92:690-8.

34. Mitrani-Rosenbaum S, Tsvieli R, Tur-Kaspa R. Oestrogen stimulates differential transcription of human papillomavirus type 16 in SiHa cervical carcinoma cells. J Gen Virol 1989;70:2227-32.

35. Rousseau M, Franco E, Villa L, et al. A cumulative case-control study of risk factor profiles for oncogenic and nononcogenic cervical human papillomavirus infections. Cancer Epidemiol Biomarkers Prev 2000;9:469-76.

36. Chan P, Chang A, Cheung J, et al. Determinants of cervical human papillomavirus infection: differences between high- and low-oncogenic risk types. J Infect Dis 2002;185:28-35.

37. Kjaer S, van den Brule A, Bock J, et al. Determinants for genital human papillomavirus infection in 1000 randomly chosen young Danish women with normal Pap smear. Different risk profiles for oncogenic and non-oncogenic HPV types? Cancer Epidemiol Biomarkers Prev 1997;6:799-805.

38. Richardson H, Franco E, Pintos J, Bergeron J, Arella M, Tellier P. Determinants of low- and high-risk cervical HPV infections in Montreal university students. Sex Transm Dis 2000;27:79-86.

39. Ludicke F, Stalberg A, Vassilakos P, Major A, Campana A. High- and intermediate-risk human papillomavirus infection in sexually active adolescent females. J Pediatr Adolesc Gynecol 2001;14:171-4.

40. Hogewoning CJ, Bleeker MC, van den Brule AJ, et al. Condom use promotes regression of cervical intraepithelial neoplasia and clearance of human papillomavirus: a randomized clinical trial. Int J Cancer 2003; 107:811-6.

41. Castellsague $X$, Muñoz N. Chapter 3: Cofactors in human papillomavirus carcinogenesis-Role of parity, oral contraceptives, and tobacco smoking. J Natl Cancer Inst Monogr 2003;31:20-8.
42. Poppe W, Ide P, Drijkoningen M, Lauweryns J, Van Assche F. Tobacco smoking impairs the local immunosurveillance in the uterine cervix. An immunohistochemical study. Gynecol Obstet Invest 1995;39:34-8.

43. Prokopczyk B, Cox JE, Hoffmann D, Waggoner SE. Identification of tobaccospecific carcinogen in the cervical mucus of smokers and nonsmokers. J Natl Cancer Inst 1997;89:868-73.

44. Melikian A, Wang X, Waggoner S, Hoffman D, El-Bayoumy K. Comparative response of normal and of human papillomavirus-16 immortalized human epithelial cervical cells to benzo[a ]pyrene. Oncol Rep 1999;6:1371-6.

45. Brabin L. Interactions of the female hormonal environment, susceptibility to viral infections and disease progression. AIDS Patient Care 2002;16:211-21.

46. Reichman M, Judd J, Longcope C, et al. Effects of alcohol consumption on plasma and urinary hormone concentration in premenopausal women. J Natl Cancer Inst 1993;85:722-7.

47. Hankinson S, Willett W, Manson J, et al. Alcohol, height, and adiposity in relation to estrogen and prolactin levels in postmenopausal women. J Natl Cancer Inst 1995;87:1297-302.

48. Castle PE, Giuliano AR. Chapter 4: Genital tract infections, cervical inflammation, and antioxidant nutrients-assessing their roles as human papillomavirus cofactors. J Natl Cancer Inst Monogr 2003;31:29-34.

49. Sedjo R, Inserra $P$, Abrahamsen $M$, et al. Human papillomavirus persistence and nutrients involved in the methylation pathway among a cohort of young women. Cancer Epidemiol Biomarkers Prev 2002;11:353-9.

50. Sedjo R, Roe D, Abrahamsen M, et al. Vitamin A, carotenoids and risk of persistent oncogenic human papillomavirus infection. Cancer Epidemiol Biomarkers Prev 2002;11:876-84.

51. Giuliano AR, Siegel EM, Roe DJ, et al. Dietary intake and risk of persistent human papillomavirus (HPV) infection: the Ludwig-McGill HPV natural history study. J Infect Dis 2003;188:1508-16.

52. Zhang S, Hunter DJ, Hankinson SE, et al. A prospective study of folate intake and the risk of breast cancer. JAMA 1999;281:1632-7.

53. Moscicki A, Hills N, Shiboski S, et al. Risks for incident human papillomavirus infection and low-grade squamous intraepithelial lesion development in young females. JAMA 2001;285:2995-3002. 